\title{
¿Cuántas personas fuman? Percepción del nivel de consumo y su relación con el consumo de cigarrillos en adolescentes chilenos
}

'Escuela de Psicología, Pontificia Universidad Católica de Chile. ${ }^{2} S$ chool of Population Health, University of Auckland, New Zealand. aPhD, Psicóloga. bPhD Candidate.

Estudio financiado por Proyecto FONDECYT \#1090563.

Fondecyt no influyó en el diseño, el análisis, resultados ni en las conclusiones de este trabajo.

Recibido el 20 de agosto de 2011, aceptado el 27 de febrero de 2012.

Correspondencia a: Paula Repetto.

Avenida Vicuña Mackenna

4860, Macul, Santiago

Escuela de Psicología.

Teléfono: 3547666 Fax: 5533092

E-mail: prepetto@uc.cl

\author{
PAULA REPETTO ${ }^{1, a}$, MARGARITA BERNALES ${ }^{2, b}$
}

\section{Perception of smoking rates and its relationship with cigarette use among Chilean adolescents}

Background: Cigarette smoking among adolescents is a major health problem in Chile, which is determined by several factors. Among these, it is important the role of perceived norms regarding cigarette use among peers and the general population. Aim: To study the social norms about the perceived prevalence of smoking and its relationship with cigarette smoking among Chilean adolescents. Material and Methods: Participants were 480 adolescents of educational institutions from the South-East area of the Metropolitan Region, who completed a questionnaire that included questions regarding cigarette use and the perceived prevalence of cigarette use among peers and adults. Results: Adolescents overestimated the percentage of smokers compared to national statistics regarding their peers and adult population. The perception of adolescents regarding the rates of use among peers predicted both current and future use. Conclusions: Cigarette smoking is influenced by perceived norms regarding use and, despite the current smoking restrictions in place, youth continue to perceive that smoking is a common behavior.

(Rev Med Chile 2012; 140: 740-745).

Key words: Adolescent; Ethical theory; Smoking.
E 1 consumo de cigarrillos en jóvenes es un importante problema de salud importante en Chile ${ }^{1}$. Las estadísticas obtenidas por CONACE sugieren que, si bien éste ha disminuido durante las últimas décadas, $33 \%$ de los escolares fuma de manera habitual ${ }^{2}$. Otras estadísticas, como las obtenidas a través de la última Encuesta Nacional de Salud ${ }^{3}$ muestran que $40,6 \%$ de la población adulta fuma de manera habitual (44,2\% la prevalencia de hombres y $37,1 \%$ la de mujeres). Estas estadísticas indican que fumar cigarrillos es un problema de salud que continúa siendo importante en Chile. La preocupación por el consumo de cigarrillos en la población chilena llevó a las autoridades a implementar cambios legislativos como los definidos por la Ley 19.4194, los cuales están orientados a restringir el consumo de cigarrillos en lugares públicos, evitar que los menores de edad estén expuestos al humo del cigarrillo y prohibir la venta de cigarrillos a menores de edad. Si bien estas medidas han ayudado a disminuir el consumo de cigarrillos, las estadísticas chilenas muestran que los adolescentes continúan fumando en niveles más altos comparados con adolescentes de otros países del mundo ${ }^{5}$. Por esta razón, estudios que ayuden a comprender por qué los adolescentes chilenos continúan fumando en altas tasas son importantes para poder desarrollar intervenciones más efectivas.

La investigación respecto del consumo de cigarrillos ha mostrado que la conducta de fumar cigarrillos se encuentra determinada por una multiplicidad de factores tanto de nivel social como personal. Entre los determinantes estudiados, los 
investigadores destacan la importancia de las normas sociales percibidas respecto a cuán normativa es la conducta de fumar tanto entre el grupo de pares como en la población general ${ }^{6}$. Los estudios muestran que los adolescentes suelen sobreestimar el consumo de cigarrillos tanto de pares como de adultos y que este factor predice consumo de cigarrillos actual y futuro, siendo un predictor más importante que el consumo real de la población ${ }^{7,8}$.

Los resultados de estos estudios sugieren que los adolescentes que creen que la conducta de fumar es altamente prevalente entre sus pares y en la población general se inician más temprano en el consumo, fuman más y tienen planes de iniciarse o mantenerse en el consumo en el futuro ${ }^{7,8}$. En un estudio ya clásico, Botvin y cols (1992) estudiaron longitudinalmente las normas percibidas respecto al consumo de cigarrillos en una muestra de escolares en Estados Unidos de Norteamérica ${ }^{9}$. Los resultados de su estudio mostraron que aquellos adolescentes que estimaban que $50 \%$ o más de sus pares y/o adultos fuman, fumaban más y presentaban tasas de consumo más altas dos años después, una vez realizado el seguimiento. Consistente con estos resultados, en estudios posteriores se ha mostrado de manera sistemática que aquellos adolescentes que creen que fumar cigarrillos es una conducta que ocurre en alta frecuencia tanto entre sus pares como en la población general, es más probable que fumen ${ }^{6,10}$.

Los investigadores han propuesto distintas explicaciones para dar cuenta de por qué las normas percibidas respecto de la prevalencia del consumo de cigarrillos influyen en el consumo de cigarrillos en adolescentes. En primer lugar, ellos señalan la importancia que tiene para los adolescentes adecuarse a las conductas y normas de su grupo de pares y, por lo tanto, si ellos perciben o creen que fumar es una conducta habitual y aceptable entre sus pares, es más probable que fumen ${ }^{11,12}$. Por otro lado, es posible que los adolescentes hagan inferencias respecto de la prevalencia del consumo en función de lo que ellos observan en su ambiente, y si observan que varias personas fuman, es posible que utilicen esta información para hacer estimaciones de la conducta de fumar en la población general ${ }^{6}$. Estos autores señalan que la conducta de fumar cigarrillos es muy visible y fácil de recordar. Esta gran visibilidad influirá en la percepción que los adolescentes tienen respecto de la prevalencia en el consumo independiente si están expuestos a pocos o muchos fumadores ${ }^{7}$. Otros autores proponen que esta percepción es el resultado del marketing de las empresas tabacaleras, que ha llevado a los adolescentes y, a la población general, a creer que fumar es una conducta que se da en una alta prevalencia entre sus pares y en la población general ${ }^{13}$. Finalmente, algunos investigadores señalan que estas creencias muestran lo fácil que es acceder a cigarrillos para los adolescentes. Este fácil acceso los llevaría a pensar que es muy probable que sus pares fumen y experimenten con cigarrillos 6 .

Independiente de las razones por las cuales los adolescentes fuman, los resultados muestran de manera consistente que los adolescentes tienden a sobreestimar la prevalencia del consumo entre sus pares y en la población general ${ }^{6,7}$, y que estas creencias se asocian con mayor consumo de cigarrillos en adolescentes ${ }^{10,14}$.

La influencia de las normas sociales en relación al consumo de cigarrillos ha sido poco explorada en adolescentes chilenos. En consecuencia, el presente estudio tuvo como objetivo estudiar las creencias normativas respecto de la prevalencia en el consumo de cigarrillos en adolescentes chilenos y su relación con el consumo de cigarrillos. Tal como se ha descrito en investigaciones anteriores, se esperaba encontrar que los adolescentes sobrestimarían la tasa de consumo entre pares y entre adultos y, que estas creencias se asociarían con las conductas de consumo de cigarrillos en adolescentes. Por otro lado, esperamos encontrar que las normas percibidas predecirían los planes de consumo futuro que tengan los adolescentes.

\section{Material y Métodos}

\section{Procedimiento}

Los datos utilizados para el presente estudio son parte de un estudio longitudinal cuyo objetivo ha sido examinar los determinantes de los patrones de cambio en el consumo de cigarrillos en jóvenes chilenos. Para efectos del presente estudio se usaron los datos de la primera medición que fue realizada el año 2009. El estudio fue aprobado por el Comité de Ética de la Escuela de Psicología de la Pontificia Universidad Católica de Chile y el Comité de Bioética de FONDECYT, cumpliendo así con la Declaración de Helsinki. Los participantes firmaron un asentimiento (menores de edad) o consentimiento (mayores de 18 años) informado 
para poder participar en el estudio. Los padres de los menores de edad también debieron autorizar la participación de sus hijos en el estudio.

Una vez obtenida la autorización de las instituciones educacionales involucradas, de los padres, y de los participantes, se llevó a cabo la aplicación del cuestionario. El cuestionario fue aplicado de manera colectiva en sala de clases a los participantes por evaluadores entrenados especialmente. Para efectos de este estudio sólo se incluyeron menores de edad.

\section{Instrumentos}

El cuestionario incluyó preguntas respecto al consumo de cigarrillos tomadas de la Encuesta Mundial de Tabaquismo Juvenil ${ }^{15} \mathrm{y}$ de otros estudios anteriores realizados con población chilena y en otros países. Se les preguntó a los participantes si han probado o no cigarrillos, la edad en la cual probaron, tiempo que pueden pasar sin fumar, auto-percepción como fumador y planes para fumar en el futuro.
En relación a las normas sociales, se les pidió que estimaran en porcentajes la cantidad de adultos que fuman y de jóvenes de su mismo grupo de edad. Para eso, se usaron las preguntas utilizadas en estudios anteriores con poblaciones similares ${ }^{16}$. Se incluyeron variables demográficas como edad y sexo como variables de control. Un resumen de las preguntas utilizadas se presenta en la Tabla 1.

\section{Participantes}

Los participantes en el estudio fueron 480 adolescentes de diferentes establecimientos educacionales públicos y particulares subvencionados del área Sur-Oriente de la Región Metropolitana, todos de nivel socioeconómico medio y mediobajo de acuerdo a la categorización del Ministerio de Educación ${ }^{17}$. El promedio de edad de los participantes fue de 14,97 años $(\mathrm{DE}=1,63)$ y $48 \%$ correspondió a hombres. Según curso, 12,7\% cursaba séptimo básico, $13,3 \%$ octavo, $23,3 \%$ primero medio, $19,7 \%$ segundo medio, $18,1 \%$ tercero medio y $12,9 \%$ cuarto año medio.

Tabla 1. Preguntas incluidas en el estudio y alternativas de respuesta

\begin{tabular}{|c|c|}
\hline Pregunta & Alternativas de respuesta \\
\hline $\begin{array}{l}\text { ¿Alguna vez has probado cigarrillos, aunque sea una o dos } \\
\text { fumadas o "piteadas"? }\end{array}$ & $\begin{array}{l}\text { Sí } \\
\text { No }\end{array}$ \\
\hline $\begin{array}{l}\text { ¿Cuántos años tenías cuando trataste de fumar o probaste } \\
\text { por primera vez un cigarrillo, aunque haya sido sólo una } \\
\text { piteada? }\end{array}$ & (En blanco, los participantes completan esta pregunta) \\
\hline $\begin{array}{l}\text { ¿Cómo describirías la forma en que tú fumas? (frecuencia } \\
\text { de consumo) }\end{array}$ & $\begin{array}{l}\text { No fumo } \\
\text { Sólo he probado } \\
\text { Fumo ocasionalmente } \\
\text { Fumo regularmente }\end{array}$ \\
\hline $\begin{array}{l}\text { ¿Cuánto tiempo puedes pasar sin fumar antes de sentir que } \\
\text { necesitas un cigarrillo? }\end{array}$ & $\begin{array}{l}\text { Menos de una hora } \\
\text { Entre } 1 \text { y } 3 \text { horas } \\
\text { Más de } 3 \text { horas pero menos de un día } \\
\text { Un día entero } \\
\text { Varios días } \\
\text { Una semana o más } \\
\text { No fumo }\end{array}$ \\
\hline $\begin{array}{l}\text { ¿Qué crees que pasará contigo en los próximos cinco años } \\
\text { respecto del cigarrillo? }\end{array}$ & $\begin{array}{l}\text { No estaré fumando } \\
\text { Pienso que estaré fumando igual que ahora } \\
\text { Pienso que estaré fumando más que ahora } \\
\text { Creo que habré dejado de fumar }\end{array}$ \\
\hline $\begin{array}{l}\text { Imagina que se junta un grupo de } 100 \text { personas de tu edad } \\
\text { ¿Cuántas de ellas crees tú que fuman? }\end{array}$ & (En blanco, los participantes completan esta pregunta) \\
\hline $\begin{array}{l}\text { Imagina que se junta un grupo de } 100 \text { adultos, ¿cuántos } \\
\text { crees tú que fuman? }\end{array}$ & (En blanco, los participantes completan esta pregunta) \\
\hline
\end{tabular}




\section{Plan de análisis}

El análisis se realizó en diferentes etapas. En primer lugar se realizaron análisis descriptivos y correlacionales. Esto permitió conocer la distribución de los puntajes, determinar si había errores de codificación y examinar multicolinealidad entre las variables.

En las siguientes etapas examinamos si las creencias respecto del nivel de consumo de cigarrillos en adultos y su grupo de pares predicen consumo presente y futuro, para eso llevamos a cabo un análisis de regresión en el cual ingresamos como variables de control: sexo, edad y haber probado.

\section{Resultados}

\section{Análisis descriptivos}

Entre los participantes en el estudio, 64,7\% señaló haber probado cigarrillos alguna vez en su vida. El promedio de edad en el cual probaron cigarrillos fue de 12,19 años $(\mathrm{DE}=3,91)$. En relación a la frecuencia de consumo, $52,1 \%$ se denominó como no fumador, $19,4 \%$ señaló que sólo ha probado los cigarrillos, $19,8 \%$ se autodenominó como fumador ocasional y $8,8 \%$ como fumador habitual. Entre los participantes 1,2\% señaló que puede pasar sin fumar menos de una hora, $0,4 \%$ entre 1 y 3 horas, $1,4 \%$ más de 3 horas pero menos de un día, 2,7\% puede pasar un día sin fumar, $7,0 \%$ una varios días pero menos de una semana, $15,9 \%$ puede pasar sin fumar una semana o más. El resto de los participantes (71,2\%), se declaró no fumador. Al preguntar respecto de los planes para consumir en los próximos 5 años, 57,6\% señaló que no estará fumando, $3,9 \%$ que estará fumando igual que ahora, $6,5 \%$ estará fumando más que ahora y $12,9 \%$ habrá dejado de fumar.

Al examinar las normas sociales relacionadas con el consumo, encontramos que, en promedio, los adolescentes piensan que $65,20 \%(\mathrm{DE}=22,87)$ de sus pares fuma y que 78,46\% $(\mathrm{DE}=20,16)$ de los adultos. Encontramos diferencias significativas en las creencias respecto al porcentaje de pares que fuman dependiendo si han probado cigarrillos $\left(\mathrm{t}_{435}=-5,739, \mathrm{p}<0,01\right)$. No encontramos diferencias significativas respecto de las normas percibidas respecto del consumo de cigarrillos en adultos cuando comparamos adolescentes que han probado cigarrillos con aquellos que no han probado $\left(\mathrm{t}_{438}=-, 734, \mathrm{NS}\right)$.

\section{Análisis de regresión}

En la siguiente etapa examinamos si las normas percibidas predicen consumo actual y futuro. Encontramos que la percepción de los adolescentes respecto de la frecuencia de consumo de sus pares predice consumo actual y futuro, aun cuando controlamos por la variable "haber probado cigarrillos alguna vez en su vida". También encontramos que el tiempo que los adolescentes pueden pasar sin fumar, se relaciona de manera inversa con la percepción de la frecuencia del consumo de pares y de adultos, de modo que mientras menos tiempo pueden pasar sin fumar creen que un porcentaje mayor de sus pares y adultos fuma. Los resultados de los análisis de regresión se resumen en Tabla 2.

\section{Conclusiones}

El presente estudio tuvo como objetivo examinar las normas percibidas respecto al consumo de cigarrillos en adolescentes chilenos y su relación con el consumo de cigarrillos. Consistente con los resultados descritos en estudios anteriores, encontramos que los adolescentes sobreestimaron el porcentaje de personas que fuman comparado con las tasas poblacionales, tanto en relación a sus pares como respecto de la población adulta, esto es si consideramos que la cifra actual de consumo de cigarrillos en Chile es de 40,6\% en adultos ${ }^{3} y$ en escolares es de $33 \%{ }^{2}$. Nuestros resultados sugieren que, en esta muestra de adolescentes, esta estimación es mucho más alta que las prevalencias de consumo nacionales, elemento que podría contribuir a explicar por qué los adolescentes chilenos continúan fumando en altas tasas a pesar de los cambios legislativos implementados.

También encontramos que las creencias respecto a la prevalencia percibidas del consumo entre pares predice frecuencia de consumo actual, tiempo que cada adolescente pasa sin necesidad de fumar y planes para fumar en el futuro. Estos resultados son consistentes con propuestas que sugieren que las normas sociales respecto del consumo de cigarrillos entre pares, es una importante influencia en el consumo. Si bien no encontramos, tal como esperábamos, que las normas percibidas sobre el consumo de cigarrillos en adultos se asocie con frecuencia de consumo y con consumo futuro, este resultado es consistente con los obtenidos en investigaciones anteriores ${ }^{9}$. Nuestros resultados sugieren que para los adolescentes, puede tener 
Tabla 2. Resultados Modelos de Regresión

\begin{tabular}{|c|c|c|c|c|}
\hline Modelo & Variables & $\beta$ & $\mathbf{t}$ & $\mathbf{R}^{2}$ (Modelo) \\
\hline \multirow[t]{6}{*}{ Frecuencia consumo } & Constante & & $2,16^{*}$ & $0,32^{* *}$ \\
\hline & Sexo & 0,02 & 0,42 & \\
\hline & Edad & $-0,09$ & $-0,19$ & \\
\hline & Ha probado cigarrillos & 0,54 & $12,22 * *$ & \\
\hline & Cuántos fuman de 100 personas de su edad & 0,10 & $2,03^{*}$ & \\
\hline & Cuántos fuman de 100 adultos & $-0,08$ & $-1,76$ & \\
\hline \multirow{6}{*}{$\begin{array}{l}\text { Tiempo que puede pasar sin } \\
\text { necesidad de fumar }\end{array}$} & Constante & & 10,69 & $0,12^{* *}$ \\
\hline & Sexo & $-0,02$ & $-0,45$ & \\
\hline & Edad & $-0,02$ & $-0,32$ & \\
\hline & Ha probado cigarrillos & 2,70 & $-5,22 * *$ & \\
\hline & Cuántos fuman de 100 personas de su edad & $-0,13$ & $-2,33^{*}$ & \\
\hline & Cuántos fuman de 100 adultos & $-0,01$ & $-0,14^{* *}$ & \\
\hline \multirow[t]{6}{*}{ Planes para fumar en 5 años } & Constante & & $2,16^{*}$ & $0,33^{* *}$ \\
\hline & Sexo & 0,018 & 0,417 & \\
\hline & Edad & $-0,09$ & $-0,190$ & \\
\hline & Ha probado cigarrillos & 0,541 & $12,22 * *$ & \\
\hline & Cuántos fuman de 100 personas de su edad & 0,10 & $2,03^{*}$ & \\
\hline & Cuántos fuman de 100 adultos & $-0,08$ & $-1,76$ & \\
\hline
\end{tabular}

Se presentan los resultados del modelo de regresión para cada medición de consumo, los valores de $\beta$ para cada predictor y el valor de t y su significación. Los valores de significación menores al $p<0,01$ se representan con ** y los valores entre 0,01 pero menores al valor de $p<0,05$ se representan con *. En la última columna se incluyen los valores de $\mathrm{R}^{2}$ ajustado para cada modelo.

una mayor importancia el consumo de cigarrillos de sus pares para explicar el consumo actual y futuro comparado con el consumo de los adultos de su entorno, influencia que puede ser explicada por características evolutivas de esta población ${ }^{8}$. Sin embargo, para comprender mejor cómo se construyen estas creencias y se mantienen el tiempo, así como para profundizar en las diferencias de influencia de las normas sociales de consumo de pares y adultos son necesarias nuevas investigaciones.

Los resultados de este estudio tienen importantes implicancias para las políticas de salud en Chile. Por un lado, van en la línea de los potenciales cambios legislativos que están orientados a restringir aún más los lugares de consumo. Los estudios muestran que la implementación de cambios legislativos que disminuyan la exposición a personas que fuman pueden contribuir a la disminución en el consumo de jóvenes en la medida que esta conducta se haga cada vez menos visible y, por lo tanto, percibida como menos común ${ }^{18-21}$. Por otro lado, estos resultados sugieren que implementar intervenciones que ayuden a corregir estas normas percibidas entre los adolescentes chilenos, tal como se han llevado a cabo en otras partes del mundo, puede ayudar a disminuir el consumo ${ }^{21,22}$.

Finalmente, hay algunas limitaciones que son importantes de señalar. En primer lugar, dado que este estudio no es longitudinal, es posible que la asociación entre consumo y creencias respecto al nivel de consumo reflejen más bien una justificación respecto a la propia conducta de fumar. Por lo tanto, aquellos adolescentes que fuman, van a señalar que hay más fumadores que aquellos que no fuman. En segundo lugar, la muestra incluyó a adolescentes de nivel socioeconómico medio y medio-bajo, lo cual no nos permite concluir respecto de la población general de adolescentes chilenos. A pesar de estas limitaciones, es importante señalar que los resultados son similares a los descritos en investigaciones anteriores que muestran la importancia de estas creencias para explicar consumo de cigarrillos en jóvenes. Estos resultados pueden constituir una contribución adicional para la comprensión del consumo de 
cigarrillos en adolescentes chilenos. Futuros estudios, deberían explorar cómo estas creencias cambian y examinar las explicaciones que los autores han propuesto para dar cuenta de cómo estas creencias se construyen.

\section{Referencias}

1. Caris L, Varas M, Anthony C, Anthony J. Behavioral problems and tobacco use among adolescents in Chile. Rev Panam Salud Publica 2003; 14 (2): 84-90.

2. Consejo Nacional para el Control de Estupefacientes CONACE. Octavo Estudio Nacional de Drogas en Población Escolar de Chile. 2009; Available from: http:// www.conacedrogas.cl/portal/index.php?option $=$ com_c ontent $\&$ view $=$ article\&id $=282 \&$ Itemid $=368$.

3. Ministerio de Salud MINSAL. Encuesta Nacional de Salud Chile 2009-2010. 2011; Available from: http://www. redsalud.gov.cl/portal/url/item/99c12b89738d80d5e040 01011e0113f8.pdf.

4. Subsecretaría de Salud Pública. Ministerio de Salud. República de Chile. Ley N 19.419. Materias relativas al consumo del tabaco. Diario Oficial de la República de Chile.

5. Warren C, Lea V, Lee J, Jones N, Asma S, McKenna M. Change in tobacco use among 13-15 year olds between 1999 and 2008: findings from the Global Youth Tobacco Survey. Glob Health Promot 2009; 16 (2 Suppl): 38-90.

6. Unger J, Rohrbach L. Why do Adolescents Overestimate Their Peers' Smoking Prevalence? Correlates of Prevalence Estimates Among California 8th-Grade Students. Journal of Youth and Adolescence 2002; 31 (2): 147-53.

7. Eisenberg M, Forster J. Adolescent smoking behavior: measures of social norms. Am J Prev Med 2003; 25 (2): 122-8.

8. Reid JL, Manske SR, Leatherdale ST. Factors related to adolescents' estimation of peer smoking prevalence. Health Educ Res 2008; 23 (1): 81-93.

9. Botvin GJ, Botvin EM, Baker E, Dusenbury L, Goldberg CJ. The false consensus effect: predicting adolescents' tobacco use from normative expectations. Psychol Rep 1992; 70 (1): 171-8.

10. Rhodes N, Roskos-Ewoldsen D, Edison A, Bradford M. Attitude and norm accessibility affect processing of antismoking messages. Health Psychol 2008; 27 (3 Suppl): 224-32.
11. Steinberg L. Adolescence. Fifth ed. New York: McGrawHill; 1999.

12. Hammond D, Fong G, Zanna M, Thrasher J, Borland R. Tobacco denormalization and industry beliefs among smokers from four countries. Am J Prev Med 2006; 31 (3): 225-32.

13. Brown A, Moodie C. The influence of tobacco marketing on adolescent smoking intentions via normative beliefs. Health Educ Res 2009; 24 (4): 721-33.

14. Olds R, Thombs D. The relationship of adolescent perceptions of peer norms and parent involvement to cigarette and alcohol use. J Sch Health 2001; 71 (6): 223 8.

15. World Health Organization WHO. Global Youth Tobacco Survey (GYTS): Available from: http://www.cdc.gov/ tobacco/global/gyts/methodology.htm.

16. Primack BA, Switzer GE, Dalton MA. Improving measurement of normative beliefs involving smoking among adolescents. Arch Pediatr Adolesc Med 2007; 161 (5): 434-9.

17. MINEDUC. Ficha Establecimientos Escolares. Santiago: MINEDUC; 2011 [cited 2011 20-06-2011]; Available from: http://infoescuela.mineduc.cl/FichaEstablecimiento/Busqueda.

18. Albers AB, Siegel M, Cheng DM, Rigotti NA, Biener L. Effects of restaurant and bar smoking regulations on exposure to environmental tobacco smoke among Massachusetts adults. Am J Public Health 2004; 94 (11): 1959-64.

19. Orbell S, Lidierth P, Henderson CJ, Geeraert N, Uller C, Uskul AK, et al. Social-cognitive beliefs, alcohol, and tobacco use: a prospective community study of change following a ban on smoking in public places. Health Psychol 2009; 28 (6): 753-61.

20. Botello-Harbaum MT, Haynie DL, Iannotti RJ, Wang J, Gase L, Simons-Morton B. Tobacco control policy and adolescent cigarette smoking status in the United States. Nicotine Tob Res 2009; 11 (7): 875-85.

21. Hamilton WL, Biener L, Brennan RT. Do local tobacco regulations influence perceived smoking norms? Evidence from adult and youth surveys in Massachusetts. Health Educ Res 2008; 23 (4): 709-22.

22. Hancock L, Henry N. Perceptions, Norms and Tobacco Use in College Residence Hall Freshmen: Evaluation of a Social Norms Marketing Intervention. Perkins HW, editor. San Francisco: Jossey-Bass; 2003. 